Internat. J. Math. \& Math. Sci.

Vol. 23, No. 10 (2000) 663-673

S0161171200001757

(C) Hindawi Publishing Corp.

\title{
STABILITY OF A CHARACTERIZATION OF NORMAL DISTRIBUTIONS BASED ON THE FIRST TWO CONDITIONAL MOMENTS
}

\author{
TRUC T. NGUYEN and KHOAN T. DINH
}

(Received 23 March 1998 and in revised form 27 July 1998)

\begin{abstract}
A characterization of normal distributions of two independent random variables $X$ and $Y$ with a finite $E\left[X^{2}\right]$ based on the linearity of $E[X \mid X+Y]$ and the homoscedasticity of $\operatorname{var}[X \mid X+Y]$ given by Rao (1976) is proved to be stable.

Keywords and phrases. Conditional density function, normal close, characteristic function, small parameter.
\end{abstract}

2000 Mathematics Subject Classification. Primary 62E10.

1. Introduction and basic results. The problem of characterization of normal distributions in empirical Bayes view based on the linearity of the posterior mean and the homoscedasticity of the posterior variance was studied by several authors $[2,4,6]$.

Let $U_{1}, \ldots, U_{n}$ be a random sample from the distribution $F(u \mid \theta)$ such that $E\left[U_{j} \mid\right.$ $\theta]=\theta$ exists, $\theta$ is a location parameter of $F(u \mid \theta)$, then the observed values of $U_{j}$ can be written as $u_{j}=\theta+w_{j}, j=1, \ldots, n$, where $w_{j}$ does not depend on $\theta$, and is the observed values of a random sample $W_{1}, \ldots, W_{n}$ from some distribution function. Assume that $\theta$ is a given value of an unobserved random variable $\Theta$, then $\Theta$ and the random sample $W_{1}, \ldots, W_{n}$ are independent. Set $Z=\sum_{j=1}^{n} U_{j} / n$ and $X=\sum_{j=1}^{n} W_{j} / n$, then $Z=\Theta+X$, where $\Theta$ and $X$ are independent. It is clear that to study the normality of the random variables $\Theta$ and $W$ is equivalent to study the normality of the random variables $\Theta$ and $X$. Then, without loss of generality, we can assume that $n=1$ in the random sample $U_{1}, \ldots, U_{n}$. Assumed the linearity of the posterior mean $E[\Theta \mid Z=z]$ with respect to $z$, and the homoscedasticity of the posterior variance $\operatorname{var}[\Theta \mid Z=z]$, Rao (1976) gave the following result on the normality of $Y_{1}$ and $\Theta$.

Let $X$ and $Y$ be two independent random variables having the nondegenerate distributions $F$ and $G$, respectively. Set $Z=X+Y$, then the following characterization of normality of $F$ and $G$ were obtained.

THEOREM 1.1. Suppose that $E\left[X^{2}\right]$ is finite. The following conditions:

$$
E[X \mid Z]=a_{o}+a_{1} Z, \quad \operatorname{var}[X \mid Z]=b_{o},
$$

where $0<a_{1}<1$, and $a_{o}, a_{1}, b_{0}$ are constants, hold if and only if both $F$ and $G$ are normal distributions.

The following result characterizes normality of a distribution $F$ based on a random sample from $F$, and is obtained directly from Theorem 1.1. 
COROLLARY 1.2. Let $X_{1}, \ldots, X_{n}, n>1$, be a random sample from a nondegenerate distribution $F$, and let $Z=\sum_{j=1}^{n} X_{j}$. The condition

$$
\operatorname{var}\left[X_{1} \mid Z\right]=b_{o},
$$

holds if and only if $F$ is a normal distribution.

DEFINITION 1.3. A distribution $F$ is $\varepsilon$-normal if there exist $\mu \in R$ and $\sigma>0$ such that $\sup _{X}|F(x)-\Phi((X-\mu) / \sigma)| \leq \varepsilon$, where $\Phi$ is the distribution function of a standard normal distribution.

Let a distribution $F$ be characterized by a property $S$. This characterization of $F$ based on $S$ is stable if the property $S$ is replaced by a weaker property $S_{\varepsilon}$, where $\varepsilon$ is a "small parameter", then the distribution $F_{\varepsilon}$ is close to $F$ in term of $\varepsilon$ [5]. Sapogov [7] showed the stability of Cramer's theorem, that is, with some supplementary conditions, he showed that if $F=F_{1} * F_{2}$ is $\varepsilon$-normal, then $F_{1}$ is $C \sigma_{F_{1}}{ }^{-3}(\ln (1 / \varepsilon))^{-1 / 2}$. normal, and $F_{2}$ is $C \sigma_{F_{2}}^{-3}(\ln (1 / \varepsilon))^{-1 / 2}$-normal. Hoang [3], with supplementary conditions, showed that $\varepsilon$-independence of $X+Y$ and $X-Y$, where $X$ and $Y$ are independent, implies $\beta_{1}(\varepsilon)$-normality of $F_{1}$ and $F_{2}$, and he also showed that $\varepsilon$-independence of $\bar{X}=(1 / n) \sum_{j=1}^{n} X_{j}$ and $S^{2}=\sum_{j=1}^{n}\left(X_{j}-\bar{X}\right)^{2}$, where $X_{1}, \ldots, X_{n}$ are i.i.d. $F$, implies $\beta_{2}(\varepsilon)$ normality of $F$. In his results, $\beta_{1}(\varepsilon)$ and $\beta_{2}(\varepsilon)$ both are of the order of $(\ln (1 / \varepsilon))^{-1 / 2}$. In this paper, we show the stability of the characterizations of normal distributions given by Theorem 1.1 and Corollary 1.2. To show these stability results, we need the following result on a measure of closeness of two distribution functions using their characteristic functions given by Esseen [1]. (See also [5]).

THEOREM 1.4. Let $F$ and $G$ be distribution functions and $\varphi$ and $\psi$ be their respective characteristic functions. If $G$ has the derivative $G^{\prime}$, then

$$
\sup _{X}|F(x)-G(x)| \leq \frac{1}{\Pi} \int_{-T}^{T}\left|\frac{\varphi(t)-\psi(t)}{t}\right| d t+\frac{24}{\Pi T} \sup _{X}\left|G^{\prime}(x)\right| .
$$

In the proofs of the theorems in Section 2 and Section 3 below, the inequality

$$
\left|e^{z}-1\right| \leq|z| e^{|z|},
$$

for any complex number $z$, is used several times.

2. Stability of the characterization given by Theorem 1.1. In this section, we show that the characterization of normal distributions given by Theorem 1.1 is stable, that is, we show that if the posterior mean is $\varepsilon$-linear and the posterior mean is $\varepsilon$-homoscedastic, then $F$ and $G$ both are $C[\ln (1 / \varepsilon)]^{-1 / 2}$-normal.

THEOREM 2.1. Let $X$ and $Y$ be two independent random variables with nondegenerate distribution functions $F$ and $G$, respectively. Assume that $E\left[X^{2}\right]$ is finite and set $Z=X+Y$. If

$$
\begin{aligned}
E[X \mid Z] & =a_{o}+a_{1} Z+b_{1}(Z), \\
\operatorname{var}[X \mid Z] & =b_{o}+b_{2}(Z),
\end{aligned}
$$


where $a_{1}, b_{o}$ are constants, $0<a_{1}<1$. Assume that $\sup _{Z}\left|b_{1}(Z)\right|, \sup _{Z}\left|b_{2}(Z)\right|<\varepsilon$, and $\varepsilon<e^{-2\left(b_{o}+2 \sqrt{\sigma_{X}{ }^{2}+\sigma_{Y}{ }^{2}}+5\right) /\left(1-a_{1}\right)}$, where $\sigma_{X}{ }^{2}$ and $\sigma_{Y}{ }^{2}$ are the variances of $X$ and $Y$, respectively, then both $F$ and $G$ are $C(\ln (1 / \varepsilon))^{-1 / 2}$-normal, where $C$ depends on $a_{1}, b_{o}, \sigma_{X}^{2}$, and $\sigma_{Y}^{2}$.

Before proving Theorem 2.1, the following question on the set of the distributions $F$ and $G$ is raised: does the set of the distributions $F$ and $G$ that satisfy (2.1) and (2.2) contain only normal distributions? In other words, is there any $F$ and $G$ such that $Z$ is not normal and (2.1) and (2.2) hold? The answer to this question is given by the following example.

EXAMPLE 2.2. Suppose that $X$ has an $N(0,1)$ distribution and $Y$ has an $(1 / 2) N(0,1)$ $+(1 / 2) N(0,1-\varepsilon)$ distribution, $0<\varepsilon<1$, are two independent random variables. Then $Z=X+Y$ has an $(1 / 2) N(0,2)+(1 / 2) N(0,2-\varepsilon)$ distribution. Hence, $X \mid Z=z$ has an $\alpha N(z / 2,1 / 2)+(1-\alpha) N(z /(2-\varepsilon),(1-\varepsilon) /(2-\varepsilon))$ distribution, where

$$
\alpha=\frac{1 / \sqrt{2} e^{-x^{2} / 4}}{1 / \sqrt{2} e^{-x^{2} / 4}+1 / \sqrt{2-\varepsilon} e^{-x^{2} / 2(2-\varepsilon)}} .
$$

Therefore, $E[X \mid Z=z]=z / 2+b_{1}(z)$, where

$$
b_{1}(z)=\frac{\varepsilon z}{\sqrt{2}(2-\varepsilon)\left[\sqrt{2}+\sqrt{2-\varepsilon} e^{\varepsilon z^{2} / 4(2-\varepsilon)}\right]} .
$$

Hence,

$$
\left|b_{1}(z)\right|<\varepsilon|z| e^{-\varepsilon Z^{2} / 4}<\varepsilon, \quad \operatorname{var}[X \mid Z=z]=\frac{1}{2}+b_{2}(z),
$$

where

$$
b_{2}(z)=\frac{-\varepsilon\left[4 \sqrt{2}(2-\varepsilon)^{1 / 2} e^{-z^{2} /(2-\varepsilon)}+\left(2(2-\varepsilon)+z^{2}\right) e^{-(4-\varepsilon) z^{2} / 4(2-\varepsilon)}\right]}{4 \sqrt{2}(2-\varepsilon)^{2} e^{-z^{2} / 2(2-\varepsilon)}+4(2-\varepsilon)^{5 / 2} e^{-z^{2} / 4}} .
$$

Then $\operatorname{var}[X \mid Z=z]<1 / 2$ and

$$
\left|b_{2}(z)\right|<\frac{\varepsilon\left(12+z^{2}\right) e^{-(4-\varepsilon) z^{2} / 4(2-\varepsilon)}}{4 \sqrt{2} e^{-z^{2} / 2(2-\varepsilon)}}=\frac{\varepsilon\left(12+z^{2}\right) e^{-z^{2} / 4}}{4 \sqrt{2}}<3 \sqrt{2} \frac{\varepsilon}{2} .
$$

Proof OF TheOrem 2.1. Assume that $\varepsilon<1$. By (2.1) and a finite $E\left[X^{2}\right], E\left[Y^{2}\right]$ is also finite and $b_{o}^{2}>0$. Without loss of generality, assume that $E[X]=E[Y]=0$, and $E[\operatorname{var}[X \mid Z]]=b_{o}$. This implies $a_{o}=0, E\left[b_{1}(Z)\right]=E\left[b_{2}(Z)\right]=0$ and (2.1) and (2.2) can be written as

$$
\begin{gathered}
E[X \mid Z]=a_{1} Z+b_{1}(Z), \\
E\left[X^{2} \mid Z\right]=b_{o}+a_{1}^{2} Z^{2}+2 a_{1} Z b_{1}(Z)+b_{1}^{2}(Z)+b_{2}(Z) .
\end{gathered}
$$

Multiply both sides of (2.8) by $e^{i t Z}$ and take the expected values. The following equations in $\varphi$ and $\psi$, the characteristic functions of $F$ and $G$, respectively, are obtained.

$$
\begin{gathered}
\left(1-a_{1}\right) \varphi^{\prime}(t) \psi(t)=a_{1} \varphi(t) \psi^{\prime}(t)+S_{1}(t), \\
\varphi^{\prime \prime}(t) \psi(t)=-b_{o} \varphi(t) \psi(t)+a_{1}^{2} \varphi^{\prime \prime}(t) \psi(t)+2 a_{1}^{2} \varphi^{\prime}(t) \psi^{\prime}(t)+a_{1}^{2} \varphi(t) \psi^{\prime \prime}(t)+S_{2}(t),
\end{gathered}
$$


where

$$
S_{1}(t)=E\left[i e^{i t Z} b_{1}(Z)\right], \quad S_{2}(t)=E\left[-e^{i t Z}\left(2 a_{1} Z b_{1}(Z)+b_{1}^{2}(Z)+b_{2}(Z)\right)\right] .
$$

Hence, for any $t$,

$$
\left|S_{1}(t)\right|=\left|E\left[i e^{i t Z} b_{1}(Z)\right]\right| \leq E\left[\left|b_{1}(Z)\right|\right] \leq \varepsilon .
$$

By Cauchy-Schwarz inequality

$$
\begin{aligned}
\left|E\left[e^{i t Z} Z b_{1}(Z)\right]\right| & \leq E\left[|Z|\left|b_{1}(Z)\right|\right] \leq\left(E\left[Z^{2}\right]\right)^{1 / 2}\left(E\left[b_{1}^{2}(Z)\right]\right)^{1 / 2} \leq \sqrt{\sigma_{X}^{2}+\sigma_{Y}^{2}} \varepsilon, \\
\left|S_{2}(t)\right| & =\left|E\left[-e^{i t Z}\left(2 a_{1} Z b_{1}(Z)+b_{1}^{2}(Z)+b_{2}(Z)\right)\right]\right| \\
& \leq 2\left|a_{1}\right|\left|E\left[Z b_{1}(Z)\right]\right|+E\left[b_{1}^{2}(Z)\right]+\left|E\left[b_{2}(Z)\right]\right| \\
& \leq 2 a_{1} \sqrt{\sigma_{X}^{2}+\sigma_{Y}^{2}} \varepsilon+\varepsilon^{2}+\varepsilon \leq 2\left(a_{1} \sqrt{\sigma_{X}^{2}+\sigma_{Y}^{2}}+1\right) \varepsilon .
\end{aligned}
$$

Since $\varphi(0)=\psi(0)=1, \varphi^{\prime}(0)=\psi^{\prime}(0)=0$, and the continuity of characteristic functions, let

$$
\delta(\varepsilon)=\sup \left\{\delta:|\varphi(t)|,|\psi(t)| \geq \varepsilon^{1 / 4}, \frac{\left|\varphi^{\prime}(t)\right|}{|\varphi(t)|}, \frac{\left|\psi^{\prime}(t)\right|}{|\psi(t)|} \leq 1,|t| \leq \delta\right\} .
$$

For $|t| \leq \delta(\varepsilon)$, divide both sides of (2.9) by $\varphi(t) \psi(t)$, the following equations are obtained

$$
\begin{gathered}
\left(1-a_{1}\right) \frac{\varphi^{\prime}(t)}{\varphi(t)}=a_{1} \frac{\psi^{\prime}(t)}{\psi(t)}+R_{1}(t), \\
\left(1-a_{1}^{2}\right) \frac{\varphi^{\prime \prime}(t)}{\varphi(t)}=-b_{o}+a_{1}^{2} \frac{\psi^{\prime \prime}(t)}{\psi(t)}+2 a_{1}^{2}\left(\frac{\varphi^{\prime}(t)}{\varphi(t)}\right)\left(\frac{\psi^{\prime}(t)}{\psi(t)}\right)+R_{2}(t),
\end{gathered}
$$

where

$$
R_{1}(t)=\frac{S_{1}(t)}{\varphi(t) \psi(t)}, \quad R_{2}(t)=\frac{S_{2}(t)}{\varphi(t) \psi(t)}
$$

From (2.11)

$$
\left|R_{1}(t)\right|=\frac{\left|S_{1}(t)\right|}{|\varphi(t) \| \psi(t)|} \leq \frac{\varepsilon}{\varepsilon^{1 / 4} \varepsilon^{1 / 4}}=\varepsilon^{1 / 2}, \quad R_{1}(0)=S_{1}(0)=0,
$$

and from (2.12)

$$
\left|R_{2}(t)\right|=\frac{\left|S_{2}(t)\right|}{|\varphi(t) \| \psi(t)|} \leq \frac{2 a_{1}\left(\sqrt{\sigma_{X}^{2}+\sigma_{Y}{ }^{2}}+1\right) \varepsilon}{\varepsilon^{1 / 4} \varepsilon^{1 / 4}}=2 a_{1}\left(\sqrt{\sigma_{X}^{2}+\sigma_{Y}^{2}}+1\right) \varepsilon^{1 / 2} .
$$

Set

$$
h(t)=\frac{\varphi^{\prime}(t)}{\varphi(t)}, \quad k(t)=\frac{\psi^{\prime}(t)}{\psi(t)} .
$$


Then

$$
\frac{\varphi^{\prime \prime}(t)}{\varphi(t)}=h^{\prime}(t)+h^{2}(t), \quad \frac{\psi^{\prime \prime}(t)}{\psi(t)}=k^{\prime}(t)+k^{2}(t)
$$

From (2.14),

$$
k(t)=\frac{1-a_{1}}{a_{1}} h(t)-\frac{R_{1}(t)}{a_{1}}
$$

and then,

$$
k^{\prime}(t)=\frac{1-a_{1}}{a_{1}} h^{\prime}(t)-\frac{R_{1}^{\prime}(t)}{a_{1}} .
$$

Substitute in (2.15) the following equation is obtained

$$
h^{\prime}(t)=-\frac{b_{o}}{1-a_{1}}-\frac{a_{1}^{2}}{1-a_{1}}\left[\frac{R_{1}^{\prime}(t)}{a_{1}}+\frac{R_{1}^{2}(t)}{a_{1}^{1}}+\frac{2\left(1-2 a_{1}\right)}{a_{1}^{2}} R_{1}(t) h(t)\right]+\frac{R_{2}(t)}{1-a_{1}} .
$$

From $E[X]=0$,

$$
\begin{aligned}
h(t)=\frac{\varphi^{\prime}(t)}{\varphi(t)}= & -\frac{b_{o}}{1-a_{1}} t+\frac{1}{1-a_{1}} \\
& \times \int_{0}^{t}\left[-a_{1}^{2}\left(\frac{R_{1}^{\prime}(u)}{a_{1}}+\frac{R_{1}^{2}(u)}{a_{1}^{2}}+\frac{2\left(1-2 a_{1}\right)}{a_{1}^{2}} R_{1}(u) h(u)\right)+R_{2}(u)\right] d u,
\end{aligned}
$$

and from $\varphi(0)=1$,

$$
\begin{aligned}
\varphi(t)= & \exp \left\{-\frac{b_{o}}{2\left(1-a_{1}\right)} t^{2}+\frac{1}{1-a_{1}}\right. \\
& \left.\times \int_{0}^{t} \int_{0}^{v}\left[-a_{1}^{2}\left(\frac{R_{1}^{\prime}(u)}{a_{1}}+\frac{R_{1}^{2}(u)}{a_{1}^{2}}+\frac{2\left(1-2 a_{1}\right)}{a_{1}^{2}} R_{1}(u) h(u)\right)+R_{2}(u)\right] d u d v\right\} \\
= & \exp \left\{-\frac{b_{o}}{2\left(1-a_{1}\right)} t^{2}+R(t)\right\},
\end{aligned}
$$

where

$$
R(t)=\frac{1}{1-a_{1}} \int_{0}^{t} \int_{0}^{v}\left[-a_{1}^{2}\left(\frac{R_{1}^{\prime}(u)}{a_{1}}+\frac{R_{1}(u)^{2}}{a_{1}^{2}}+\frac{2\left(1-2 a_{1}\right)}{a_{1}^{2}} R_{1}(u) h(u)\right)+R_{2}(u)\right] d u d v .
$$


For

$$
\begin{aligned}
|t| \leq & \delta(\varepsilon),|\operatorname{Re}\{R(t)\}| \\
\leq & \mid\left\{-\frac{a_{1}}{1-a_{1}} \int_{0}^{t} R_{1}(v) d v\right. \\
& \left.\quad+\frac{1}{1-a_{1}} \int_{0}^{t} \int_{0}^{v}\left[R_{2}(u)-R_{1}^{2}(u)-2\left(1-2 a_{1}\right) R_{1}(u) h(u)\right] d u d v\right\} \mid \\
\leq & \left|\frac{a_{1}}{1-a_{1}} \int_{0}^{t} R_{1}(v) d v\right| \\
& +\left|\frac{1}{1-a_{1}} \int_{0}^{t} \int_{0}^{v}\left[\left|R_{2}(u)\right|+R_{1}^{2}(u)+2\left|1-2 a_{1}\left\|R_{1}(u)\right\| h(u)\right|\right] d u d v\right| \\
\leq & \frac{a_{1}}{1-a_{1}} \varepsilon^{1 / 2}|t|+\frac{1}{1-a_{1}}\left[2\left(a_{1} \sqrt{\sigma_{X}^{2}+\sigma_{Y}^{2}}+1\right) \varepsilon^{1 / 2}+\varepsilon+2\left|1-2 a_{1}\right| \varepsilon^{1 / 2}\right] \frac{|t|^{2}}{2} \\
\leq & \frac{\varepsilon^{1 / 2}}{1-a_{1}}\left[a_{1}|t|+\frac{1}{2}\left(2 a_{1} \sqrt{\sigma_{X}^{2}+\sigma_{Y}^{2}}+1+2\left|1-2 a_{1}\right|\right)|t|^{2}\right] \\
\leq & \frac{\varepsilon^{1 / 2}}{1-a_{1}}\left[|t|+\frac{1}{2}\left(2 \sqrt{\sigma_{X}^{2}+\sigma_{Y}^{2}}+3\right)|t|^{2}\right] .
\end{aligned}
$$

Hence,

$$
\begin{aligned}
&|\varphi(t)|=\exp \{-\frac{b_{o}}{2\left(1-a_{1}\right)}|t|^{2} \\
&+ \operatorname{Re}\left\{-\frac{a_{1}}{1-a_{1}} \int_{0}^{t} R_{1}(v) d v+\frac{1}{1-a_{1}}\right. \\
& \quad\left.\left.\quad \times \int_{0}^{t} \int_{0}^{v}\left[R_{2}(u)-R_{1}^{2}(u)-2\left(1-2 a_{1}\right) R_{1}(u) h(u)\right] d u d v\right\}\right\} \\
& \geq \exp \left\{-\frac{b_{o}}{2\left(1-a_{1}\right)}|t|^{2}-\frac{\varepsilon^{1 / 2}}{1-a_{1}}\left[|t|+\frac{1}{2}\left(2 \sqrt{\sigma_{X^{2}}+\sigma_{Y}^{2}}+3\right)|t|^{2}\right]\right\} .
\end{aligned}
$$

Since the function

$$
\exp \left\{-\frac{b_{o}}{2\left(1-a_{1}\right)}|t|^{2}-\frac{\varepsilon^{1 / 2}}{1-a_{1}}\left[|t|+\frac{1}{2}\left(2 \sqrt{\sigma_{X}^{2}+\sigma_{Y}^{2}}+3\right)|t|^{2}\right]\right\}
$$

is decreasing in $|t|$, and $|\varphi(t)|>\varepsilon^{1 / 4}$ for $|t| \leq \delta(\varepsilon), \delta(\varepsilon)$ is not smaller than the solution $t_{0}>0$ of the equation

$$
\exp \left\{-\frac{b_{o}}{2\left(1-a_{1}\right)}|t|^{2}-\frac{\varepsilon^{1 / 2}}{1-a_{1}} \mid\left[|t|+\frac{1}{2}\left(2 \sqrt{\sigma_{X}^{2}+\sigma_{Y}^{2}}+3\right)|t|^{2}\right]\right\}=\varepsilon^{1 / 4}
$$

or equivalently, the equation

$$
\left[b_{o}+\varepsilon^{1 / 2}\left(2 \sqrt{\sigma_{X}^{2}+\sigma_{Y}^{2}}+3\right)\right]|t|^{2}+2 \varepsilon^{1 / 2}|t|=\frac{1-a_{1}}{2} \ln \left(\frac{1}{\varepsilon}\right) .
$$

The function on the left-hand side of (2.31) is an even function, and for $t>0$, it is increasing. Then equation (2.31) has two opposite solutions. For $t>0$, equation (2.31) becomes

$$
\left[b_{o}+\varepsilon^{1 / 2}\left(2 \sqrt{\sigma_{X}^{2}+\sigma_{Y}^{2}}+3\right)\right] t^{2}+2 \varepsilon^{1 / 2} t=\frac{1-a_{1}}{2} \ln \left(\frac{1}{\varepsilon}\right) .
$$


For $t>1$,

$$
\begin{aligned}
{\left[b_{o}+\varepsilon^{1 / 2}\left(2 \sqrt{\sigma_{X}^{2}+\sigma_{Y}^{2}}+5\right)\right] t } & \leq\left[b_{o}+\varepsilon^{1 / 2}\left(2 \sqrt{\sigma_{X}^{2}+\sigma_{Y}^{2}}+3\right)\right] t^{2}+2 \varepsilon^{1 / 2} t \\
& \leq\left[b_{o}+\varepsilon^{1 / 2}\left(2 \sqrt{\sigma_{X}^{2}+\sigma_{Y}^{2}}+5\right)\right] t^{2}
\end{aligned}
$$

and for $t \leq 1$,

$$
\begin{aligned}
{\left[b_{o}+\varepsilon^{1 / 2}\left(2 \sqrt{\sigma_{X}^{2}+\sigma_{Y}^{2}}+5\right)\right] t^{2} } & \leq\left[b_{o}+\varepsilon^{1 / 2}\left(2 \sqrt{\sigma_{X}^{2}+\sigma_{Y}^{2}}+3\right)\right] t^{2}+2 \varepsilon^{1 / 2} t \\
& \leq\left[b_{o}+\varepsilon^{1 / 2}\left(2 \sqrt{\sigma_{X}^{2}+\sigma_{Y}^{2}}+5\right)\right] t
\end{aligned}
$$

Hence,

$$
t_{o} \geq \min \left\{\frac{\left(1-a_{1}\right) \ln (1 / \varepsilon)}{2\left[b_{o}+\varepsilon^{1 / 2}\left(2 \sqrt{\sigma_{X}^{2}+\sigma_{Y}^{2}}+5\right)^{1 / 2}\right]}, \frac{\left(1-a_{1}\right) \ln (1 / \varepsilon)}{2\left[b_{o}+\varepsilon^{1 / 2}\left(2 \sqrt{\sigma_{X}{ }^{2}+\sigma_{Y}}+5\right)\right]}\right\} .
$$

If we choose $\varepsilon<e^{-2\left(b_{o}+2 \sqrt{\sigma_{X}^{2}+\sigma_{Y}^{2}}+5\right) /\left(1-a_{1}\right)}$, then

$$
\frac{\left(1-a_{1}\right)}{2\left[b_{o}+2 \sqrt{\sigma_{X}{ }^{2}+\sigma_{Y}^{2}}+5\right]}>1, \quad \delta(\varepsilon) \geq t_{o} \geq \delta_{o}(\varepsilon)=\left(\frac{\left(1-a_{1}\right) \ln (1 / \varepsilon)}{2\left[b_{o}+2 \sqrt{\sigma_{X}{ }^{2}+\sigma_{Y}{ }^{2}}+5\right]}\right)^{1 / 2} .
$$

Using [1, Theorem 1.2], where $G$ is the cumulative distribution function of a normal distribution with zero mean and the variance $b_{0} /\left(1-a_{1}\right)$, and by choosing $T=\delta_{o}(\varepsilon)$,

$$
\begin{aligned}
\sup _{X} \mid & F(x)-\Phi\left(\frac{x}{\sqrt{b_{o} /\left(1-a_{1}\right)}}\right) \mid \\
\leq & \frac{1}{\Pi} \int_{-\delta_{o}(\varepsilon)}^{\delta_{o}(\varepsilon)} \frac{\left|\varphi(t)-e^{-\left[b_{o} / 2\left(1-a_{1}\right)\right] t^{2}}\right|}{|t|} d t+\frac{24}{\Pi \delta_{o}(\varepsilon)} \sup _{X}\left|\Phi^{\prime}\left(\frac{x}{\sqrt{b_{o} /\left(1-a_{1}\right)}}\right)\right| \\
= & \frac{1}{\Pi} \int_{-\delta_{o}(\varepsilon)}^{\delta_{o}(\varepsilon)} e^{-\left[b_{o} / 2\left(1-a_{1}\right)\right] t^{2}} \frac{\left|1-e^{R(t)}\right|}{|t|} d t+\frac{12 \sqrt{2\left(1-a_{1}\right) / b_{o}}}{\Pi^{3 / 2} \delta_{o}(\varepsilon)} \\
\leq & \frac{1}{\Pi} \int_{-\delta_{o}(\varepsilon)}^{\delta_{o}(\varepsilon)} e^{-\left[b_{o} / 2\left(1-a_{1}\right)\right] t^{2}} \frac{|R(t)| e^{|R(t)|}}{|t|} d t+\frac{12 \sqrt{2\left(1-a_{1}\right) / b_{o}}}{\Pi^{3 / 2} \delta_{o}(\varepsilon)} \\
\leq & \frac{2}{\Pi} \int_{0}^{\delta_{o}(\varepsilon)} \frac{\varepsilon^{1 / 2}\left[t+\frac{1}{2}\left(2 \sqrt{\sigma_{X}^{2}+\sigma_{Y}^{2}}+3\right) t^{2}\right]}{\left(1-a_{1}\right) t} \\
& \times e^{\left(\left[-b_{o}+\varepsilon^{1 / 2}\left(2 \sqrt{\sigma_{X}^{2}+\sigma_{Y}^{2}}+3\right)\right] t^{2}+2 \varepsilon^{1 / 2} t\right) / 2\left(1-a_{1}\right)} d t+\frac{12 \sqrt{2}}{b_{o}^{1 / 2} \Pi^{3 / 2} \delta_{o}(\varepsilon)}
\end{aligned}
$$


by using (2.27),

$$
\begin{aligned}
& \leq \frac{1}{\Pi} \int_{0}^{\delta_{o}(\varepsilon)} \frac{\varepsilon^{1 / 2}\left[2+\left(2 \sqrt{\sigma_{X}^{2}+\sigma_{Y}^{2}}+3\right) t\right]}{1-a_{1}} \\
& \times e^{\left[\left(-b_{o}+2 \varepsilon^{1 / 2} \sqrt{\sigma_{X}^{2}+\sigma_{Y}{ }^{2}}+3\right) t^{2}+2 t\right] / 2\left(1-a_{1}\right)} d t+\frac{12 \sqrt{2}}{b_{o}^{1 / 2} \Pi^{3 / 2} \delta_{o}(\varepsilon)} \\
& \leq \frac{1}{\Pi} e^{\left[\left(-b_{o}+2 \varepsilon^{1 / 2} \sqrt{\sigma_{X}^{2}+\sigma_{Y}^{2}}+3\right) \delta_{o}(\varepsilon)^{2}+2 \delta_{o}(\varepsilon)\right] / 2\left(1-a_{1}\right)} \\
& \times \int_{0}^{\delta_{o}(\varepsilon)} \frac{\varepsilon^{1 / 2}\left[2+\left(2 \sqrt{\sigma_{X}^{2}+\sigma_{Y}^{2}}+3\right) t\right]}{1-a_{1}} d t+\frac{12 \sqrt{2}}{b_{o}^{1 / 2} \Pi^{3 / 2} \delta_{o}(\varepsilon)} \\
& =\frac{\varepsilon^{1 / 2}\left[4 \delta_{o}(\varepsilon)+\left(2 \sqrt{\sigma_{X}^{2}+\sigma_{Y}^{2}}+3\right) \delta_{o}(\varepsilon)^{2}\right]}{2 \Pi\left(1-a_{1}\right)} \\
& \times e^{\left[\left(-b_{o}+2 \varepsilon^{1 / 2} \sqrt{\sigma_{X}^{2}+\sigma_{Y}{ }^{2}}+3\right) \delta_{o}(\varepsilon)^{2}+2 \delta_{o}(\varepsilon)\right] / 2\left(1-a_{1}\right)}+\frac{12 \sqrt{2}}{b_{o} \Pi^{3 / 2} \delta_{o}(\varepsilon)} \\
& \leq \frac{\varepsilon^{1 / 2} \delta_{o}(\varepsilon)^{2}\left(7+2 \sqrt{\sigma_{X}^{2}+\sigma_{Y}^{2}}\right)}{2 \Pi\left(1-a_{1}\right)} \\
& \times e^{\left[-b_{o}+2 \varepsilon^{1 / 2} \delta_{o}(\varepsilon)^{2}\left(7+2 \sqrt{\sigma_{X}^{2}+\sigma_{Y}^{2}}\right)\right] / 2\left(1-a_{1}\right)}+\frac{12 \sqrt{2}}{b_{o} \Pi^{3 / 2} \delta_{o}(\varepsilon)} \\
& =\frac{\varepsilon^{1 / 2} \ln (1 / \varepsilon)\left(7+2 \sqrt{\sigma_{X}^{2}+\sigma_{Y}^{2}}\right)}{4 \Pi\left(b_{o}+2 \sqrt{\sigma_{X}^{2}+\sigma_{Y}^{2}}+5\right)} e^{b_{o} / 2\left(1-a_{1}\right)} \\
& \times e^{\left[\varepsilon^{1 / 2} \ln (1 / \varepsilon)\left(7+2 \sqrt{\sigma_{X}^{2}+\sigma_{Y}^{2}}\right)\right] / 4\left(b_{o}+2 \sqrt{\sigma_{X}^{2}+\sigma_{Y}^{2}}+5\right)} \\
& +\frac{24\left(b_{o}+2 \sqrt{\sigma_{X}^{2}+\sigma_{Y}^{2}}+5\right)}{\left(1-a_{1}\right) b_{o} \Pi^{3 / 2}}\left[\ln \left(\frac{1}{\varepsilon}\right)\right]^{-1 / 2} \\
& \leq\left\{\frac{7+2 \sqrt{\sigma_{X}^{2}+\sigma_{Y}^{2}}}{4 \Pi\left(b_{o}+2 \sqrt{\sigma_{X}^{2}+\sigma_{Y}^{2}}+5\right)} e^{\left[b_{o} / 2\left(1-a_{1}\right)\right]+\left[\left(7+2 \sqrt{\sigma_{X}^{2}+\sigma_{Y}{ }^{2}}\right) / 4\left(b_{o}+2 \sqrt{\sigma_{X}^{2}+\sigma_{Y}{ }^{2}}+5\right)\right]}\right. \\
& \left.+\frac{24\left(b_{o}+2 \sqrt{\sigma_{X}^{2}+\sigma_{Y}^{2}}+5\right)}{\left(1-a_{1}\right) b_{o} \Pi^{3 / 2}}\right\}\left[\ln \left(\frac{1}{\varepsilon}\right)\right]^{-1 / 2},
\end{aligned}
$$

since for small $\varepsilon, \varepsilon^{1 / 2} \ln (1 / \varepsilon)<1$, and $\varepsilon^{1 / 2} \ln (1 / \varepsilon)<[\ln (1 / \varepsilon)]^{-1 / 2}$. Therefore $F$ is $C[\ln (1 / \varepsilon)]^{-1 / 2}$-normal, where $C$ is a constant depends only on $a_{1}, b_{o}, \sigma_{X}{ }^{2}$ and $\sigma_{Y}{ }^{2}$. From $Z=X+Y, X=Z-Y$, substitute in (2.1) and (2.2), the following conditions on $Y$ are obtained:

$$
E[Y \mid Z]=-a_{o}+\left(1-a_{1}\right) Z-b_{1}(Z), \quad \operatorname{var}(Y \mid Z)=b_{o}+b_{2}(Z) .
$$

Similarly, $G$ is also $C[\ln (1 / \varepsilon)]^{-1 / 2}$-normal. This completes the proof.

3. Stability of the characterization given by Corollary 1.1. In this section, we show that the characterization of normal distribution given in Corollary 1.2 is stable.

THEOREM 3.1. Let $X_{1}, \ldots, X_{n}, n>1$, be a random sample from a nondegenerate distribution function $F$ with a finite second moment, and let $Z=\sum_{j=1}^{n} X_{j}$. If

$$
\operatorname{var}\left(X_{1} \mid Z\right)=\frac{n-1}{n} b_{o}+c(n, Z)
$$


where $b_{o}>0$ is a constant not depending on $Z$, $\sup _{Z}|(n /(n-1)) c(n, Z)| \leq \varepsilon^{n}, \varepsilon<$ $\min \left\{1, b_{o}^{2 / n}\right\}$, then $F$ is $C[\ln (1 / \varepsilon)]^{-1 / 2}$-normal.

Proof. Without loss of generality, assume that $E\left[\operatorname{var}\left(X_{1} \mid Z\right)\right]=((n-1) / n) b_{o}$. Hence, $E[c(n, Z)]=0$. To simplify the notation in the proof, assume that $\sup _{Z}|c(n, Z)|$ $\leq \varepsilon$, and $\varepsilon<1$. From (3.1),

$$
E\left[X_{1}^{2} \mid Z\right]=\frac{Z^{2}}{n^{2}}+\frac{n-1}{n} b_{o}+c(n, Z) .
$$

Multiply both sides of (3.2) by $e^{i t Z}$ and take the expected value, the following equation in the characteristic function $\varphi$ of $F$ is obtained:

$$
\varphi^{\prime \prime}(t) \varphi^{n-1}(t)=\varphi^{\prime 2}(t) \varphi^{n-2}(t)-b_{o} \varphi^{n}(t)+S_{n}(t),
$$

where

$$
S_{n}(t)=-\frac{n}{n-1} E\left[e^{i t Z} c(n, Z)\right]
$$

Then

$$
\sup _{t}\left|S_{n}(t)\right| \leq \frac{n}{n-1} E\left[\left|e^{i t Z}\right||c(n, Z)|\right] \leq \frac{n}{n-1} E[|c(n, Z)|] \leq \varepsilon .
$$

Let $\delta(\varepsilon)=\sup \left\{\tau:|\varphi(t)|>\varepsilon^{1 / 2 n},|t|<\tau\right\}$. Then for $|t|<\delta(\varepsilon)$, equation (3.3) can be written as

$$
\frac{\varphi^{\prime \prime}(t) \varphi(t)-\varphi^{\prime 2}(t)}{\varphi^{2}(t)}=-b_{o}+\frac{S_{n}(t)}{\varphi^{n}(t)} .
$$

Take integration both sides of (3.6) with respect to $t$, and assuming that the mean of $F$ is $\mu$,

$$
\frac{\varphi^{\prime}(t)}{\varphi(t)}=-b_{o} t+i \mu+\int_{0}^{t} \frac{S_{n}(u)}{\varphi^{n}(u)} d u .
$$

Take integration both sides of (3.7) with respect to $t$, together with the condition $\varphi(0)=1$, we get

$$
\varphi(t)=e^{-(1 / 2) b_{o} t^{2}}+i \mu t+\int_{0}^{t} \int_{0}^{v} \frac{S_{n}(t)}{\varphi^{n}(u)} d u d v
$$

Let

$$
\begin{gathered}
R_{n}(t)=\int_{0}^{t} \int_{0}^{v} \frac{S_{n}(u)}{\varphi^{n}(u)} d u d v . \\
\left|\operatorname{Re}\left(R_{n}(t)\right)\right| \leq\left|\int_{0}^{t} \int_{0}^{v} \frac{\left|S_{n}(u)\right|}{\left|\varphi^{n}(u)\right|} d u d v\right| \leq\left|\int_{0}^{t} \int_{0}^{v} \frac{\varepsilon}{\left(\varepsilon^{1 / 2 n}\right)^{n}} d u d v\right| \leq \varepsilon^{1 / 2} \frac{t^{2}}{2} .
\end{gathered}
$$

Hence,

$$
\begin{aligned}
|\varphi(t)| & =e^{-(1 / 2) b_{0} t^{2}+\operatorname{Re}\left(\int_{0}^{t} \int_{0}^{v}\left(\left|S_{n}(u)\right| /\left|\varphi^{n}(u)\right|\right) d u d v\right)} \\
& \geq e^{-(1 / 2) b_{0} t^{2}-(1 / 2) \varepsilon^{1 / 2} t^{2}}=e^{-(1 / 2)\left(b_{0}+\varepsilon^{1 / 2}\right) t^{2}} .
\end{aligned}
$$


Since $e^{-(1 / 2)\left(b_{o}+\varepsilon^{1 / 2}\right) t^{2}}$ is a decreasing function in $|t|$, and $|\varphi(t)|>\varepsilon^{1 / 2 n}$ for $|t|<$ $\delta(\varepsilon), \delta(\varepsilon)$ is not smaller than the maximum of the absolute values of the solutions of the equation

$$
e^{-(1 / 2)\left(b_{o}+\varepsilon^{1 / 2}\right) t^{2}}=\varepsilon^{1 / 2 n},
$$

or equivalently, the equation

$$
\left(b_{o}+\varepsilon^{1 / 2}\right) t^{2}=\frac{1}{n} \ln \left(\frac{1}{\varepsilon}\right) .
$$

Equation (3.12) has two opposite solutions. Let

$$
t_{o}=\left(\frac{\ln (1 / \varepsilon)}{n\left(b_{o}+\varepsilon^{1 / 2}\right)}\right)^{1 / 2}
$$

be its positive solution. Then

$$
\begin{aligned}
\delta(\varepsilon) \geq t_{o} & =\left(\frac{\ln (1 / \varepsilon)}{n\left(b_{o}+\varepsilon^{1 / 2}\right)}\right)^{1 / 2} \geq \delta_{o}(\varepsilon)=\left(\frac{\ln (1 / \varepsilon)}{n\left(b_{o}+1\right)}\right)^{1 / 2}, \\
\left|\varphi(t)-e^{-(1 / 2) b_{o} t^{2}+i \mu t}\right| & =e^{-(1 / 2) b_{o} t^{2}} \times\left|e^{R} n^{(t)}-1\right| \\
& \leq e^{-(1 / 2) b_{o} t^{2}} \times\left|R_{n}(t)\right| e^{\left|R_{n}(t)\right|}, \\
& \leq \frac{1}{2} e^{(1 / 2)\left(-b_{o}+\varepsilon^{1 / 2}\right) t^{2}} \varepsilon^{1 / 2} t^{2} \leq \frac{1}{2} \varepsilon^{1 / 2} t^{2},
\end{aligned}
$$

if we assume that $\varepsilon<b_{o}^{2}$. Using [1, Theorem 1.2] once again, for any $0<T<\delta(\varepsilon)$,

$$
\begin{aligned}
\sup _{X}\left|F(x)-\Phi\left(\frac{x-\mu}{\sqrt{b_{o}}}\right)\right| & \leq \frac{1}{\Pi} \int_{-T}^{T}\left|\frac{\varphi(t)-e^{-(1 / 2) b_{o} t^{2}+i \mu t}}{t}\right| d t+\frac{24}{\Pi T} \sup _{X}\left|\Phi^{\prime}\left(\frac{x-\mu}{\sqrt{b_{o}}}\right)\right| \\
& \leq \frac{1}{\Pi} \int_{-T}^{T} \frac{1}{2} \varepsilon^{1 / 2}|t| d t+\frac{12 \sqrt{2}}{T \sqrt{b_{o} \Pi^{3 / 2}}}=\frac{\varepsilon^{1 / 2} T^{2}}{\Pi}+\frac{12 \sqrt{2}}{\Pi^{3 / 2} T \sqrt{b_{o}}} .
\end{aligned}
$$

If we choose $T=\delta_{0}(\varepsilon)$, (3.15) becomes

$$
\begin{aligned}
\sup _{X}\left|F(x)-\Phi\left(\frac{x-\mu}{\sqrt{b_{o}}}\right)\right| & \leq \frac{\varepsilon^{1 / 2} \ln (1 / \varepsilon)}{\Pi n\left(b_{o}+1\right)}+\frac{12 \sqrt{2 n\left(b_{o}+1\right)}}{\Pi^{3 / 2} \sqrt{b_{o}}}\left[\ln \left(\frac{1}{\varepsilon}\right)\right]^{-1 / 2} \\
& \leq\left[\frac{(3 / e)^{3 / 2} \sqrt{n}}{\Pi\left(b_{o}+1\right)}+\frac{12 \sqrt{2 n\left(b_{o}+1\right)}}{\Pi^{3 / 2} \sqrt{b_{o}}}\right]\left[\ln \left(\frac{1}{\varepsilon}\right)\right]^{-1 / 2},
\end{aligned}
$$

since for $\varepsilon>0, \varepsilon^{1 / 2} \ln (1 / \varepsilon) \leq(3 / e)^{3 / 2}[\ln (1 / \varepsilon)]^{-1 / 2}$. In order for the last term of the inequality (3.16) does not depend on $n$, instead of choosing $\sup _{Z}|c(n, Z)|<\varepsilon$, we choose $\sup _{Z}|c(n, Z)|<\varepsilon^{n}$, then (3.16) becomes

$$
\sup _{X}\left|F(x)-\Phi\left(\frac{x-\mu}{\sqrt{b_{o}}}\right)\right| \leq\left[\frac{(3 / e)^{3 / 2}}{\Pi\left(b_{o}+1\right)}+\frac{12 \sqrt{2\left(b_{o}+1\right)}}{\Pi^{3 / 2} \sqrt{b_{o}}}\right]\left[\ln \left(\frac{1}{\varepsilon}\right)\right]^{-1 / 2},
$$

assuming that $\varepsilon<\min \left\{1, b_{o}^{2 / n}\right\}$. Therefore, $F$ is $C[\ln (1 / \varepsilon)]^{-1 / 2}$-normal, where $C$ depends only on $b_{o}$. 
ACKNOWLEDGEMENT. We are grateful to the referees for their valuable comments which led to a substantial improvement in the presentation of this paper.

\section{REFERENCES}

[1] C. G. Esseen, Fourier analysis of distribution functions. A mathematical study of the LaplaceGaussian law, Acta Math. 77 (1945), 1-125. MR 7,312a. Zbl 060.28705.

[2] P. K. Goel and M. H. DeGroot, Only normal distributions have linear posterior expectations in linear regression, J. Amer. Statist. Assoc. 75 (1980), no. 372, 895-900. MR 82j:62001. Zbl 454.62016.

[3] H. N. Hoang, On the stability of some characteristic properties of a normal population, Teor. Verojatnost. i Primenen 13 (1968), 308-314, English translation: Theor. Probab. Appl. 13, 299-304 (1969). MR 38\#2865. Zbl 167.47404.

[4] A. M. Kagan and Ju. N. Karpov, A Bayesian formulation of the problem of estimating a location parameter, Zap. Naučn. Sem. Leningrad. Otdel. Mat. Inst. Steklov. (LOMI) 29 (1972), 62-73. MR 49\#10026. Zbl 337.62022.

[5] A. M. Kagan, Y. V. Linnik, and C. R. Rao, Characterization problems in mathematical statistics, John Wiley \& Sons, New York-London-Sydney, 1973. MR 49\#11689. Zbl 271.62002.

[6] C. R. Rao, Characterization of prior distributions and solution to a compound decision problem, Ann. Statist. 4 (1976), no. 5, 823-835. MR 54\#14180. Zbl 341.62029.

[7] N. A. Sapogov, The stability problem for a theorem of Cramér, Izvestiya Akad. Nauk SSSR. Ser. Mat. 15 (1951), 205-218. MR 13,51f. Zbl 043.13202.

Nguyen: Department of Mathematics and Statistics, Bowling Green State UniverSITY, BOWLING GREEN, OHIO 43403-0221, USA

E-mail address: tnguyen@math.bgsu.edu

Dinh: ENVIRonmental Protection AgenCy, WASHington, DC 20460, USA 


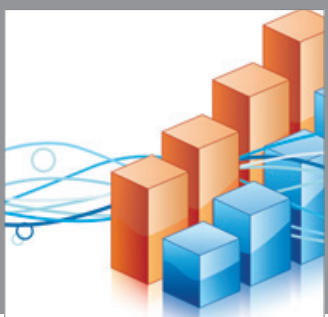

Advances in

Operations Research

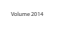

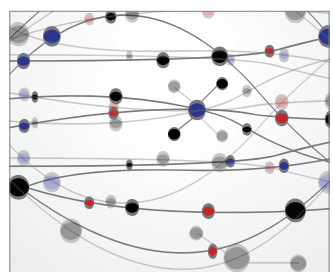

\section{The Scientific} World Journal
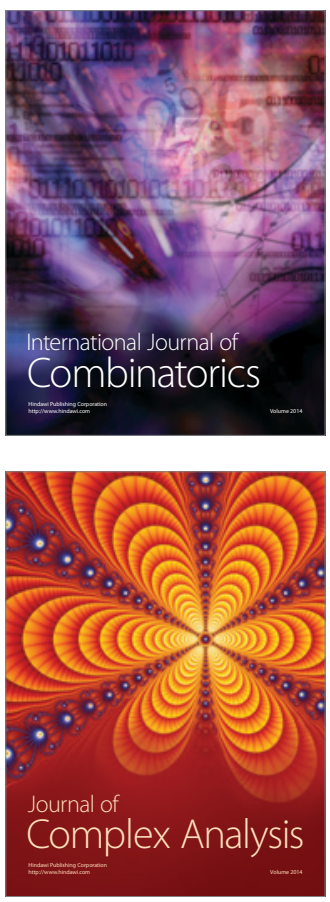

International Journal of

Mathematics and

Mathematical

Sciences
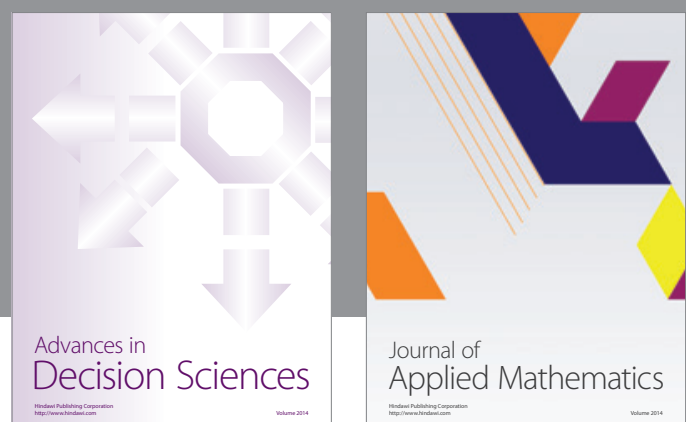

Journal of

Applied Mathematics
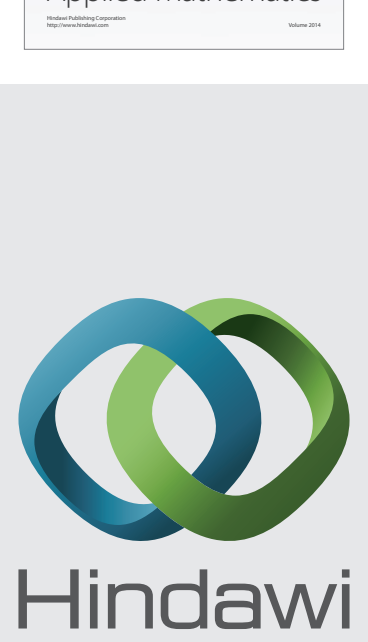

Submit your manuscripts at http://www.hindawi.com
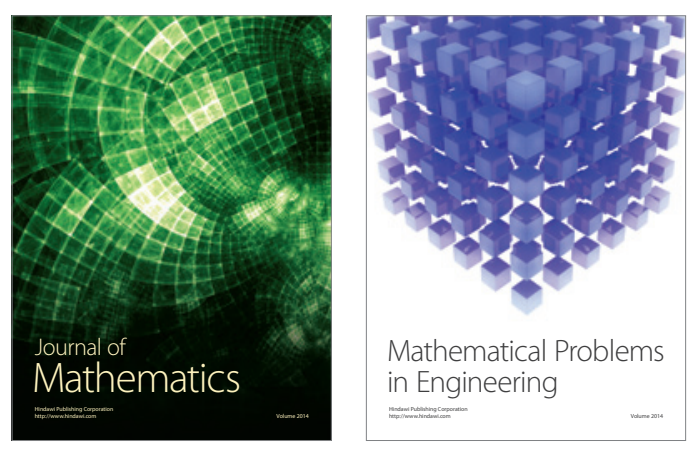

Mathematical Problems in Engineering
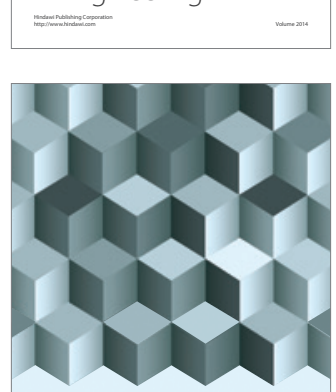

Journal of

Function Spaces
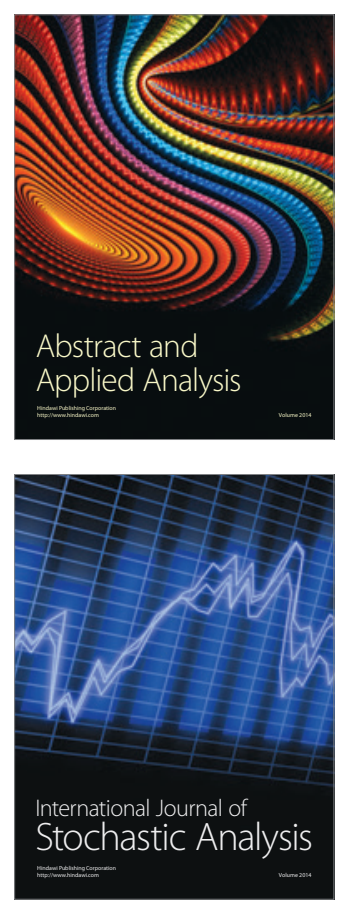

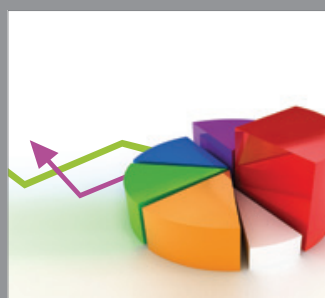

ournal of

Probability and Statistics

Promensencen
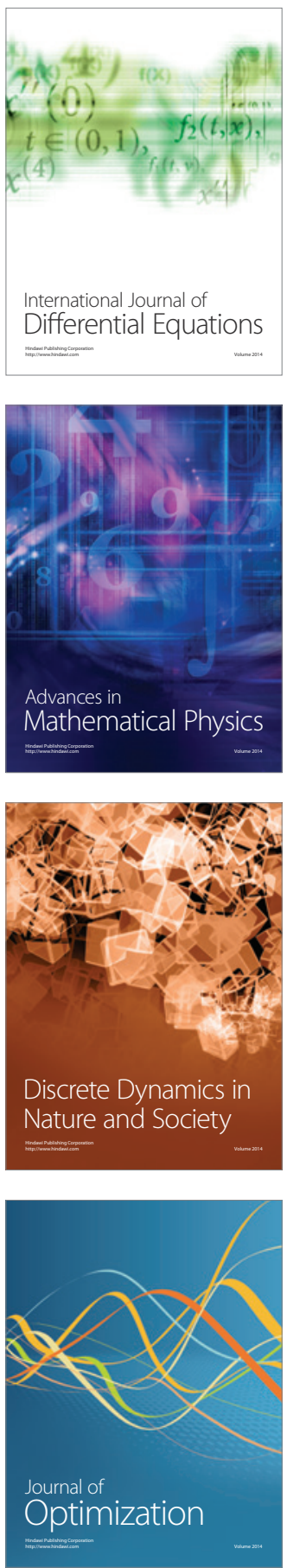